analgetics or triptans) to which they had failed to respond. Haloperidol was associated with few relapses, and in over a third of patients was reported to have interrupted a prolonged migraine attack experienced in the days leading up to the infusion, resulting in a subsequent pain-free period of days, or even weeks. Clouding these positive results, however, were the adverse effects experienced by four-fifths of patients, which were so serious in some cases that a sixth of patients said they would not like to be treated with the drug in future.

Haloperidol therefore has high efficacy in the treatment of acute migraine, but seems to be associated with more-frequent adverse effects than other neuroleptics. The authors suggest that haloperidol might best be used as a rescue medication when other treatment options have failed or cannot be used, although its adverse effects might preclude its use in some patients.

Original article Honkaniemi J et al. (2006) Haloperidol in the acute treatment of migraine: a randomized, double-blind, placebo-controlled study. Headache 46: 781-787

\section{Cerebral emboli are a potential cause of dementia}

Venous emboli entering the arterial circulation through a venous-to-arterial circulation shunt represent a possible source of spontaneous cerebral emboli (SCE), which have been associated with cerebral damage. Carotid disease is another possible source of SCE. Researchers in the UK investigated the prevalence of SCE, carotid disease and venous-to-arterial circulation shunts in patients with Alzheimer's disease (AD) or vascular dementia, compared with the prevalence in controls matched for age and sex.

In $1 \mathrm{~h}$ of transcranial Doppler monitoring, SCE were detected in 32/80 (40\%) patients with AD compared with 12/80 (15\%) controls, and in $31 / 83$ (37\%) patients with vascular dementia compared with 12/83 (14\%) controls. Adjusted for cardiovascular risk factors, the odds ratios associated with SCE were 2.70 for $A D$ and 5.36 for vascular dementia, compared with controls. Although a venousto-arterial circulation shunt indicative of a patent foramen ovale was detected in more patients with dementia than controls, the study was insufficiently powered to detect a statistically significant difference. Carotid disease was equally prevalent in patients with dementia and controls. An association was found between cardiovascular risk factors and SCE in the controls, but no such association was found in the patients with dementia.

The study detected a clear association between SCE and both $A D$ and vascular dementia, although the authors were unable to confirm the source of SCE in these conditions. SCE occurred at a similar frequency in both types of dementia, and could be a preventable or treatable cause of dementia, say the authors.

Original article Purandare N et al. (2006) Cerebral emboli as a potential cause of Alzheimer's disease and vascular dementia: case-control study. BMJ 332: 1119-1124

\section{New guidelines on prevention of a first ischemic stroke}

The American Heart Association and American Stroke Association Stroke Council recently reported new guidelines on the prevention of a first ischemic stroke. The guidelines, which are a complete revision of the last statement on this subject by the same group published in 2001, include an evidence-based classification of stroke risk factors or markers, and their potential for modification.

Goldstein et al. carried out systematic literature reviews of material published since the last statement up to January 2005. In addition, they consulted previously published guidelines, personal files and expert opinions, to bring together existing evidence and to highlight gaps in current knowledge. Recommendations were formulated using the standard American Heart Association criteria.

Many modifiable risk factors such as hypertension and diabetes were considered to be well documented, and a number of additional risk factors were classified as less well documented or potentially modifiable. These included the metabolic syndrome, alcohol and drug abuse, use of oral contraceptives, sleep-disordered breathing, migraine, elevated lipoprotein (a) levels, and inflammation or infection. A recommendation was made that aspirin should not be used for the prevention of a first stroke in males, although it was considered useful in high-risk females in whom the benefits of the treatment outweigh the risks. 